\title{
BLICKDIAGNOSE-QUIZ
}

\author{
Rezidivierendes Bluterbrechen
}

\section{Wo vermuten Sie die Blutungsquelle?}

\section{Auflösung von Seite 5}

— Primär stellte sich die Patientin erstmalig mit Teerstuhl und Bluterbrechen im Februar 2012 vor. Sowohl CT-angiografisch als auch endoskopisch konnte keine Blutungsquelle verifiziert werden. Es zeigte sich der bekannte Thoraxmagen bei kombinierter Hernie (Abb. 1a). Im stationären Verlauf kam es erneut zu einem Blutungsereignis mit Hämatemesis. Die Patientin wurde reanimationspflichtig. Nach Intubation zeigte sich bronchoskopisch schaumig-blutiges Sekret, welches als „Aspirat" bewertet wurde. In einer erneuten Ösophagogastroduodenoskopie fand sich ein großes Koagel im Fundusbereich. Vermutet wurde eine Schleimhautblutung bei kombinierter Hernienbildung und Verdacht auf Upside-down-Magen. Eine operative Versorgung wurde aufgrund des Alters und der Nebenerkrankungen der Patientin vorerst nicht empfohlen. Nach Entlassung wurde die orale Antikoagulation ambulant wieder begonnen, nun jedoch mit einer PPI-Therapie kombiniert.

Im Mai 2012 kam es erneut zu einem akuten Blutungsereignis mit Synkope, Intubations- und Transfusionspflichtigkeit. Eine neuerliche Endoskopie und Angiografie fand keine Blutungsquelle. Von chirurgischer Seite wurde eine operative Versorgung des bekannten Thoraxmagens empfohlen, welche jedoch von der Patientin abgelehnt wurde. Die Antikoagulation wurde im Konsens mit unseren internistischen Kollegen abgesetzt.

Im Juni 2012 kam es zum dritten akuten Blutungsereignis. Die Patientin wurde abermals intubations- und transfusionspflichtig. Röntgenologisch zeigten sich Aspirationsinfiltrate. Nach dem intensivmedizinischen Aufenthalt wurde einer operativen Therapie im Sinne einer Hiatoplastik mit Fundoplikatio zugestimmt. Die Operation als
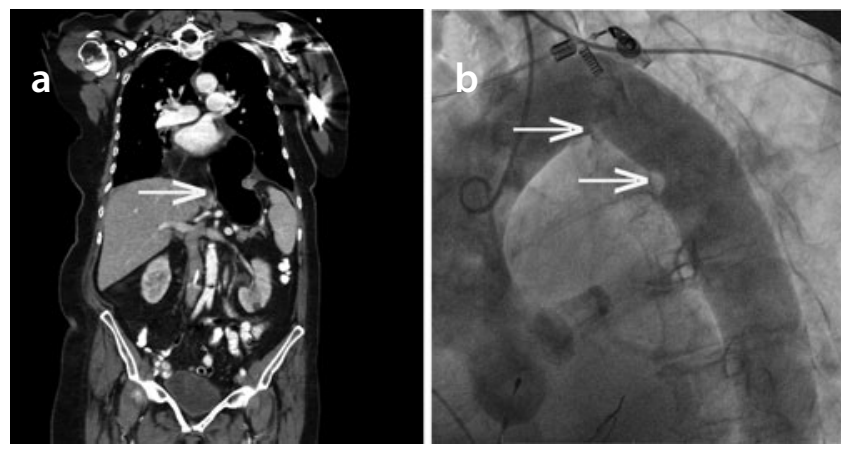

Abb. 1a CT-Schnittbild in der Frontalebene mit Darstellung eines Thoraxmagens (weißer Pfeil).

1b Angiografische Darstellung des Aortenbogens. Die weißen Pfeile zeigen die Einziehungen des Aorteninterponates.
Abb. 2a CT-Schnittbild in der Horizontalebene. Deutlich zu erkennen der zipfelige Kontrastmittelaustritt auf Höhe des distalen Aortenprothesenendes in ein sackförmig aneurysmatisches Gebilde mit Anschluss an das Bronchialsystem (siehe weißer Pfeil).

$2 \mathrm{~b}$ Angiografie des Aortenbogens. Zu erkennen die Einziehungen des Aorteninterponates. Darstellung eines Paravasates im Sinne eines Kontrastmittelaustrittes (weißer Pfeil).

2c Angiografie des Aortenbogens nach Stentversorgung.

Im Vergleich zu Abb. 2b deutliche Abnahme des Kontrastmittelaustritts.

auch der weitere Verlauf waren komplikationslos. Anfang Juni erfolgte die Entlassung.

Vier Wochen danach kam es zum erneuten Blutungsereignis. Eine
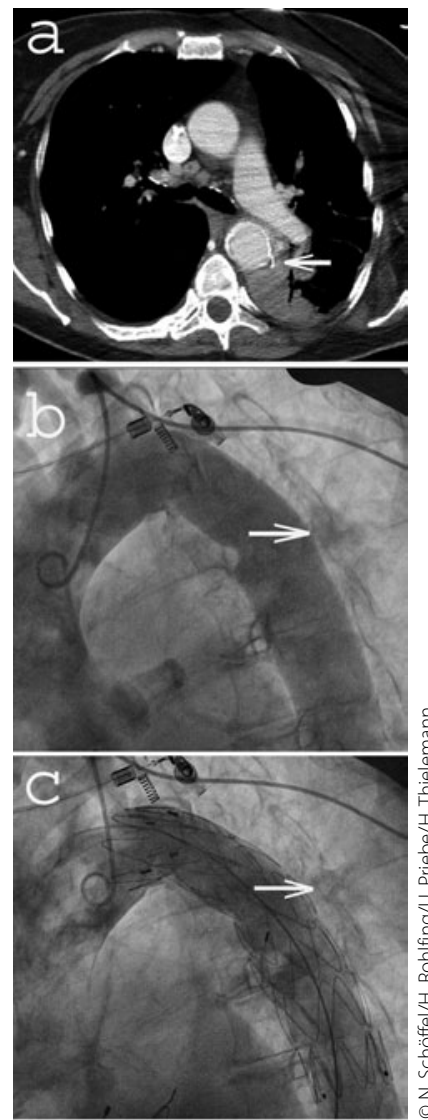
umfangreiche Notfalldiagnostik zeigte bronchoskopisch frische Blutauflagerungen. Es wurde nun differenzialdiagnostisch der Verdacht einer aorto-bronchialen Fistel bei liegender Gefäßprothese gestellt. In der durchgeführten CT-Diagnostik zeigte sich ein Kontrastmittelausstritt auf Höhe des distalen Prothesenendes mit Anschluss an das Bronchialsystem im Sinne einer aorto-bronchialen Fistel (Abb. 2a+b). Es erfolgte die Stent-Graft-Versorgung (Abb. 2c). Folgend konnte die Patientin entlassen werden. Ein erneutes Blutungsereignis trat nicht auf.

Aorto-bronchiale Fisteln sind selten, aber keine Rarität. Die dargestellte Fallkonstellation ist jedoch einzigartig. Bei einer Literaturanalyse in „pubmed“ ließ sich kein vergleichbarer Fall eruieren.

Keywords: Haematemesis - thoracic stomach - oral anticoagulation - aortobronchial fistula - aortic prosthesis

- Norman Schöffel, Henrike Rohlfing, Uwe Priebe, Henryk Thielemann Unfallkrankenhaus Berlin; Klinik für Allgemein- und Viszeralchirurgie, Warener Straße 7, D-12683 Berlin 